\title{
Dietary tryptophan depletion in humans using a simplified two amino acid formula - a pilot study
}

\author{
Maike Linden 1,2, Katrin Helmbold 1,2, Janina Kempf ${ }^{1,2,3}$, Shabnam Sippas 1,2,3, \\ Christian Filss ${ }^{4}$, Karl-Josef Langen ${ }^{4,5,6}$, Albrecht Eisert ${ }^{7}$ and Florian Daniel Zepf ${ }^{1,2,8,9 *}$ \\ 'Clinic for Child and Adolescent Psychiatry, Psychosomatics and Psychotherapy, RWTH Aachen University, \\ Aachen, Germany; ${ }^{2}$ JARA Translational Brain Medicine, Jülich Aachen Research Alliance, Jülich, Germany; ${ }^{3}$ Faculty of \\ Arts and Social Sciences, Maastricht University, Maastricht, The Netherlands; ${ }^{4}$ Section JARA-Brain, Jülich-Aachen \\ Research Alliance (JARA), Jülich, Germany; ${ }^{5}$ Institute of Neuroscience and Medicine (INM-3, -4, -5), Research Centre \\ Jülich, Jülich, Germany; 'Department of Nuclear Medicine, RWTH Aachen University Hospital, Aachen, Germany; \\ ${ }^{7}$ Department of Pharmacy, RWTH Aachen University, Aachen, Germany; ${ }^{8}$ Centre and Discipline of Child and \\ Adolescent Psychiatry, Psychosomatics and Psychotherapy, School of Psychiatry and Clinical Neurosciences \& School \\ of Paediatrics and Child Health, Faculty of Medicine, Dentistry and Health Sciences, The University of Western \\ Australia, Perth, Australia; 'Specialised Child and Adolescent Mental Health Services (CAMHS), Department of \\ Health in Western Australia, Perth, Australia
}

\section{Abstract}

Background: Acute tryptophan depletion (ATD) is a well-established dietary method in translational brain research used to briefly lower central nervous serotonin (5-hydroxytryptamine (5-HT)) synthesis. A simplified two

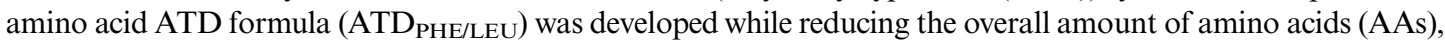
with the objective of administration especially in children and adolescents in future studies.

Objective: This study investigated tryptophan (TRP) influx rates across the blood-brain barrier (BBB) after dietary $\mathrm{ATD}_{\mathrm{PHE} / \mathrm{LEU}}$ administration relative to the ATD Moja-De protocol that has been established for use in children and adolescents.

Design: Seventy-two healthy adults (50\% females) were randomized into four groups and administered ATD

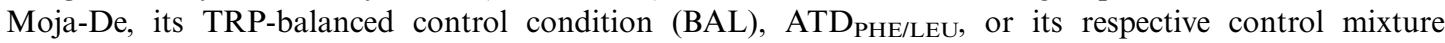
$\left(\mathrm{BAL}_{\mathrm{PHE} / \mathrm{LEU}}\right)$ in a counterbalanced, double-blind, between-subjects design. Blood samples were collected at baseline and at hourly intervals for $6 \mathrm{~h}$ after AA intake. Questionnaires about mood, taste, and challenge tolerance were completed at fixed time points.

Results: Both challenge mixtures significantly reduced central nervous TRP influx as calculated by MichaelisMenten kinetics relative to baseline and the respective control conditions with only mild and comparable side effects.

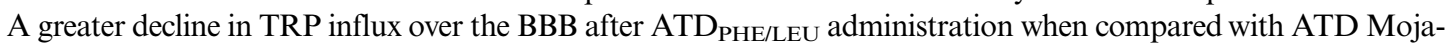
De was detected without group effects for taste, challenge tolerance, and mood. There was unintended initial short increase in plasma TRP concentrations observed after $\mathrm{ATD}_{\mathrm{PHE} / \mathrm{LEU}}$ intake, and a possible redistribution between free and protein-bound TRP triggered by protein synthesis stimulated by the ingested AAs may account for this finding. Moreover, a decline in TRP influx after BAL $\mathrm{PHE}_{\mathrm{LEU}}$ administration over a 6 - $\mathrm{h}$ period was observed, and the large amount of PHE in the BAL $\mathrm{PHE}_{\mathrm{LEU}}$ mixture may be a possible explanation for this particular phenomenon, which could have led to an unexpected increase in displacement of TRP at the BBB in this control condition.

Conclusions: This pilot study provides preliminary evidence for the possibility of lowering TRP influx as calculated by Michaelis-Menten kinetics into the brain by using a simplified ATD protocol in humans. The simplified composition of only two AAs, the lower overall AA amount, and the appropriate tolerance are characteristics of the newly developed ATD $\mathrm{PHE}_{\mathrm{LEU}}$ protocol. Future studies focusing on the effects of the ATD $\mathrm{PHE/LEU}$ protocol and its respective control condition on CSF 5-HIAA concentrations, as well as neurochemical studies in rodents, are needed to further validate this newly developed AA mixture before definite conclusions about its usability in ATDrelated research in humans, its specificity, and additional effects can be made.

Keywords: serotonin; amino acids; dietary tryptophan depletion; influx rate; Michaelis-Menten kinetics; humans

To access the supplementary material to this article, please see Supplementary files under 'Article Tools'. 
A cute tryptophan depletion (ATD) is an established dietary challenge procedure used to investigate the central nervous effects of the neurotransmitter serotonin (5-hydroxytryptamine (5-HT)) in humans and rodents by a short term and reversible reduction in the brain 5-HT synthesis rate. This method has been commonly used in translational brain research for more than 25 years and is of both clinical and scientific relevance, particularly because 5-HT modulates an extensive range of physiological and behavioral functions (1). Moreover, it is known to be involved in the pathogenesis and pathophysiology of a variety of different behaviors and neuropsychiatric disorders, including affective, eating, and attention deficit hyperactivity disorders (2-13).

ATD is accomplished through the administration of a mixture of large neutral amino acids (LNAAs) lacking tryptophan (TRP). Because 5-HT is not able to pass the blood-brain barrier (BBB), it is synthesized from its physiological precursor, the amino acid (AA) TRP, in the brain. A small proportion of TRP reaches the central nervous system through passive diffusion. The majority of free TRP uses the active transport system L-1, a carrier in the capillary cell plasma membrane specialized for the transportation of all LNAAs, to cross the BBB (14). An affinity to the aforementioned AA, increased by $100-1,000$ times, characterizes the BBB-specific type of the L-1 system (15). Moreover, L-systems differing in these and other properties can be found in all cell types in the human body (e.g. gastrointestinal tractus and hepatic and renal tissues). The uptake of TRP into the brain using L-1, which is saturated under physiological conditions, marks a facilitated diffusion that follows Michaelis-Menten kinetics with competitive substrate inhibition of the LNAAs at the common transporter (15-17). When orally administering a beverage of LNAAs lacking TRP, it was shown that TRP plasma concentrations decline because of increased protein synthesis in the liver, which is induced by the administered AA load. Based on preliminary findings in rats (18), this is thought to be an underlying principle of the ATD challenge procedure introduced by Young et al. because of reduced substrate availability for brain 5-HT synthesis with regard to reduced plasma TRP levels. Moreover, ATD makes use of the outlined competitive substrate inhibition, resulting in a diminished uptake of endogenous TRP into the central nervous system at L-1 (19-23). Specifically, the administered AAs compete with endogenous TRP at L-1 for uptake into the central nervous system. The synthesis of 5-HT in the brain comprises two steps: first, the hydroxylation of TRP by the tryptophan hydroxylase 2 (TPH2) into 5-hydroxytryptophan, followed by a 5-hydroxytryptophan decarboxylase catalysis in the second step, which leads to 5-HT $(24,25)$. ТРH2 is involved in the rate-limiting step of the central nervous 5-HT synthesis pathway, with TPH 2 showing a saturation of approximately 50\% under physiological conditions (26). As a consequence, reduced substrate availability of TRP due to competing mechanisms at the L-1 when other AAs are administered leads to a lowered central nervous 5-HT synthesis rate. As outlined above, AA administration is thought to induce restricted 5-HT synthesis by stimulating protein synthesis in the liver with the consumption of additional TRP from plasma stores $(26,27)$.

The history of ATD development is characterized by a variety of modifications of the respective AA mixtures. In 1977, Concu et al. (28) applied the ATD technique to humans using $18.2 \mathrm{~g}$ of a TRP-free mixture (containing L-glycine, L-lysine (LYS), L-methionine (MET), L-phenylalanine (PHE), L-leucine (LEU), L-isoleucine (ILE), L-threonine (THR), and L-valine (VAL)). They detected a decline in serum TRP by $42 \%$ in healthy men (29). Moja et al. (30) also tested a similar formulation, lacking glycine, in healthy male subjects. In their study, plasma TRP decreased to $\sim 35 \%$ of the initial level following the administration of an AA dose of $36.6 \mathrm{~g}$. Most of the published ATD studies refer to a protocol introduced by Young et al. (31). Young and colleagues used a $100 \mathrm{~g}$ mixture of 15 amino acids (nine LNAA and six non-essential AAs) and found a 76\% decrease in plasma TRP. This formulation can cause a large range of side effects (such as nausea, vomiting, headache, and dizziness), which were also reported in other ATD studies (2, 20, 32-35). Dougherty et al. (21) found that reducing the amino acid dose to only $50 \mathrm{~g}$ helped diminish observed side effects while implying a slightly less robust depletion when compared with the $100 \mathrm{~g}$ formulation.

ATD Moja-De, a modification of the formulation by Moja et al. (30), was developed in $2002(36,37)$ with the aim of establishing a body weight-adapted dosing regimen that can be used in minors. This concept does not only help to reduce side effects to a tolerable level but also allows the application of ATD Moja-De to children and adolescents as well as adults, opening further developmental research perspectives for future studies. Moreover, ATD Moja-De is the only available ATD formulation to date that has been shown to decrease brain 5-HT in mice (38).

Based on the above mentioned developments, we decided to establish a new formulation, called ATD PHE/ $_{\text {, }}$ LEU, for optimizing TRP depletion procedures in healthy adult humans. Once data in adult humans are obtained as proposed in this study, this formulation could potentially be used for future studies in children and adolescents. Our motivation was to introduce a new protocol for a possible later use in children and adolescents allowing a reduction of the overall AA amount and a less complex composition, leading to a solid depletion magnitude while reducing or maintaining the very low side effects established in ATD Moja-De. Hence, a new protocol was developed based on mathematical considerations and existing data 
that showed differing affinities of competing AAs to L-1 (16), thus impacting TRP influx over the BBB to

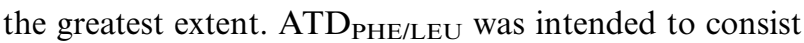
of two AAs, PHE and LEU, as these show the highest affinities to the L-1 transporting enzyme of all competing LNAAs $(16,36)$. Therefore, based on a recent study confirming the effectiveness of a similar simplified protocol in a mouse model (12), we expected adequate stimulation of protein synthesis in the liver (thus leading to a decline in plasma TRP) and a more effective displacement of TRP by PHE and LEU at the common BBB transporter.

The primary aim of this study was to make a comparison of the new dietary depletion protocol's efficacy against that of the established ATD Moja-De, contrasting challenge conditions (ATD, ATD $\mathrm{PHE}_{\mathrm{PHEU}}$ ) and their counterbalanced control conditions (BAL, BAL PHE/LEU $_{\text {) in healthy }}$ adults. We collected several blood samples over a defined time interval and calculated TRP influx rates across the BBB using Michaelis-Menten kinetics with a correction for multiple substrate inhibition.

\section{Methods}

\section{Study design}

The study employed a controlled, double-blind, betweensubjects design. One of the four different AA mixtures, ATD Moja-De, BAL (a TRP-balanced AA load serving as a control condition for ATD Moja-De), ATD PHE/LEU

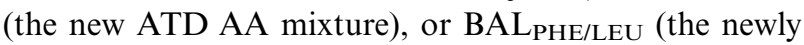
developed control condition for $\mathrm{ATD}_{\mathrm{PHE} / \mathrm{LEU}}$ ), was administered to a sample group of healthy adult subjects. Blood samples were collected at hourly intervals for $6 \mathrm{~h}$. Participants also completed questionnaires on mood, challenge tolerance, and taste evaluations.

\section{Ethics statement}

The ethics committee of the Medical Faculty of the RWTH Aachen University, Germany, evaluated and approved the study protocol. The study was carried out in accordance with the Declaration of Helsinki. All participants provided oral and written informed consent. After completion of all measurements, participants were financially compensated.

\section{Sample}

Participants were recruited through local advertisements. A total of 72 subjects ( $50 \%$ females) aged between 18 and 33 years (mean age $23.60 \pm 3.08$ years) participated in the study. All subjects were right-handed native German speakers who did not suffer from any physical (excluded by interview) or psychiatric disorder (evaluated by a standardized screening interview for psychiatric disorders, the SKIDPIT-light (39)). The exclusion criteria included the following: an IQ lower than 85; smoking; the use of regular medications excluding oral contraceptives; drug abuse; pregnancy; psychoorganic syndromes; schizophrenia; affective disorders; and neurological and somatic diseases such as migraines, asthma, diabetes, metabolic or hormonal disorders, allergies or obesity. The mean BMI of the participants was $22.4 \pm 2.5 \mathrm{~kg} / \mathrm{m}^{2}$. Using the CFT-20R (40), the participants' IQ (mean IQ $=112 \pm 13.6$ ) was assessed. Approximately 20 individuals were excluded by these criteria during the recruitment process. Primary prescreening for inclusion/exclusion criteria was conducted by telephonic interview. Written informed consent was obtained after in-person screening and clarification by a doctor. Subjects verified by self-report that they had followed the instructions regarding beverage and food intake. Negative urine drug screens and pregnancy tests were required before the administration of the AA beverages. The complete descriptive data of the sample are given in Table 1.

\section{Depletion procedure}

Measurements began at the same time each morning in order to avoid the influence of circadian rhythms on metabolism and central nervous 5-HT synthesis. The AA beverages were administered between 9:00 and 10:00 a.m. The study duration was approximately $6 \mathrm{~h}$. The participants were instructed to abstain from food high in protein beginning at 8:00 p.m. the night before each study day, and they were not allowed to drink any beverages other than water. They received a standardized low-protein breakfast (carbohydrate $68.3 \mathrm{~g}$, fat $1.13 \mathrm{~g}$, protein $5.1 \mathrm{~g}$, and dietary fiber $2.3 \mathrm{~g}$ ) containing two wheat rolls as well as one small pack of jam (25 g) and honey ( $25 \mathrm{~g})$ immediately following the administration of the AA beverages with a 20 -min time frame for breakfast. The particular AA mixtures were composed by a pharmacist and delivered within an aqueous suspension. In order to reduce the risk of undesired lump formation, the crystalline amino acids were solubilized as much as possible with a low sugar substitute in an amount of $200 \mathrm{ml}$ of water using a milk frother. Beverage intake period was reduced to a minimum of approximately $3 \mathrm{~min}$. The sample was divided into four randomized and counterbalanced groups of 18 people with equal gender distributions ( 9 women and 9 men). The administration of the different AA beverages was counterbalanced for sex. Participants of each group either received ATD, BAL, ATD PHE/LEU, or BAL $L_{\mathrm{PHE} / \mathrm{LEU} \text {. }}$

The neurodietary challenge procedure ATD Moja-De was previously used successfully with children and adolescents and had few side effects due to the administration regimen that adapted dosage to body weight $(26,36-38$, 41-44). The dosage per $10 \mathrm{~kg}$ of body weight contained L-PHE (1.32 g), L-LEU (1.32 g), L-ILE (0.84 g), L-MET $(0.5 \mathrm{~g})$, L-VAL (0.96 g), L-THR (0.6 g), and L-LYS $(0.96 \mathrm{~g})$. TRP $(0.7 \mathrm{~g}$ per $10 \mathrm{~kg}$ of body weight $)$ was added to the corresponding control condition (BAL). The newly developed AA mixture (ATD PHE/LEU $_{\text {, }}$, also dosed per 
Table 1. Descriptive data of the study sample

\begin{tabular}{|c|c|c|c|c|c|}
\hline & & ATD & BAL & ATD $_{\text {PHE/LEU }}$ & BAL \\
\hline \multirow[t]{3}{*}{ Age } & $M \pm S D$ & $23.11 \pm 2.89$ & $23.94 \pm 3.15$ & $23.67 \pm 2.66$ & $23.72 \pm 3.69$ \\
\hline & Min. & 18 & 18 & 18 & 19 \\
\hline & Max. & 30 & 32 & 29 & 33 \\
\hline \multirow[t]{3}{*}{ IQ } & $M \pm S D$ & $107.67 \pm 16.36$ & $113.22 \pm 12.10$ & $114.61 \pm 14.97$ & $112.89 \pm 10.27$ \\
\hline & Min. & 80 & 97 & 76 & 97 \\
\hline & Max. & 138 & 142 & 142 & 134 \\
\hline \multirow[t]{3}{*}{ BMI } & $M \pm S D$ & $22.90 \pm 2.53$ & $22.18 \pm 2.81$ & $22.21 \pm 2.52$ & $22.25 \pm 2.4 I$ \\
\hline & Min. & 18.8 & 17.9 & 19.4 & 18.8 \\
\hline & Max. & 27.3 & 29.0 & 29.4 & 26.9 \\
\hline
\end{tabular}

Descriptive data of the study sample are divided into four conditions/groups: acute tryptophan depletion mixture Moja-De (ATD), the TRP-balanced

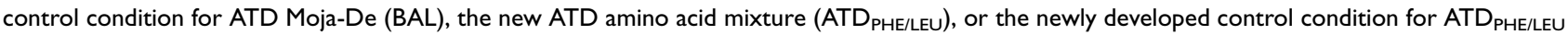
(BAL $L_{P H E / L E U}$ ). Mean \pm standard deviation $(M \pm S D)$, maximum (Max.), and minimum (Min.) values are listed for age, IQ, and body mass index (BMI).

$10 \mathrm{~kg}$ of body weight, consisted only of L-PHE $(3.2 \mathrm{~g})$ and L-LEU ( $3.0 \mathrm{~g})$; BAL $\mathrm{PHE} / \mathrm{LEU}$, the new mixture's control condition, contained an additional dose of $0.7 \mathrm{~g}$ of TRP per $10 \mathrm{~kg}$ of body weight.

\section{Blood samples}

Blood samples (BS) were collected using a permanent venous catheter at baseline $\left(T_{0}\right)$ and each hour $\left(T_{1}, T_{2}, T_{3}\right.$, $\mathrm{T}_{4}, \mathrm{~T}_{5}$, and $\mathrm{T}_{6}$ ) after the intake of ATD, BAL, ATD PHE/ LEU, or $\mathrm{BAL}_{\mathrm{PHE} / \mathrm{LEU}}\left(\mathrm{BS}_{0}, \mathrm{BS}_{1}, \mathrm{BS}_{2}, \mathrm{BS}_{3}, \mathrm{BS}_{4}, \mathrm{BS}_{5}\right.$, and $\mathrm{BS}_{6}$ ), resulting in seven blood samples per subject. A 9-ml Ethylenediaminetetraacetic acid (EDTA-Tube) (EDTA SMonovette ${ }^{\circledR}$, Sarstedt, Germany) and one 7.5-ml SerumTube (Serum S-Monovette ${ }^{\circledR}$, Sarstedt, Germany) were used for sample collection each hour. To avoid falsification of the blood values, the first $4.5 \mathrm{ml}$ of every sample was rejected. The collected blood samples were kept at room temperature for $20 \mathrm{~min}$ and centrifuged at 2,500 $\mathrm{g}$ for 10 min. The resulting clear supernatant was removed with a pipette and kept in 2-ml Eppendorf Tubes ${ }^{\circledR}$ at $-80^{\circ} \mathrm{C}$ until transportation to the laboratory.

For the TRP influx calculations, plasma levels of PHE, Tyrosine (TYR), and TRP were measured using enzymelinked immunosorbent assay (ELISA) kits (Immundiagnostik AG, Bensheim, Germany) in accordance with the manufacturer's instructions. The detection of the branchedchained AAs (BCAA: LEU, ILE and VAL) concentration using a BCAA test kit (Immundiagnostik AG, Bensheim, Germany) for photometric analyses of enzyme levels in plasma and serum was carried out according to the manufacturer's instructions. Information regarding sensitivity and specificity of the employed test kits is provided in Supplementary Table 1.

\section{Influx calculation into the brain for TRP, TYR, and PHE}

We calculated the influx of TRP across the BBB using Michaelis-Menten kinetics with a correction for multiple substrate competition, which is an established procedure in ATD-related research $(16,45)$. As the competitive sub- strate inhibition of TRP and the LNAAs at the common transporting enzyme L-1 determines the uptake of TRP into the brain, this equation provides a valid mathematical model for calculating the unidirectional TRP influx rates into the central nervous system $(26,36,46)$. The equation (see Supplementary file) includes both components influencing TRP uptake: the facilitated diffusion of AAs at L-1 and passive diffusion (36). Influx rates of the competing LNAAs PHE, TYR, as well as BCAA influx were calculated using the same equation. In addition to the abovementioned influx calculations, we also provided the following ratios to ensure comparability with previous ATD studies: [TRP]/[CAA], [TYR]/[CAA], [PHE]/[CAA], and $[\mathrm{PHE}+\mathrm{TYR}] /[\mathrm{BCAA}+\mathrm{TRP}] \quad(\mathrm{CAA}=$ competing amino acids). These ratios predict the entry rates of the involved amino acids into the brain and present another possible approach for evaluating ATD protocols.

\section{Questionnaires}

Mood

The German version of the Positive and Negative Affect Schedule (PANAS) (47) containing 10 items for positive (PA) and 10 items for negative (NA) affect was used to measure the extent to which depletion influenced the subjects' mood state. Participants rated the intensity of each item on a five-point Likert scale (from $1=$ not at all to $5=$ very much) at baseline and every hour before the collection of the blood samples.

\section{Challenge tolerance}

We developed an in-house questionnaire on the tolerance of the AA challenges to detect if the newly developed mixture caused an equal amount of side effects as the established ATD Moja-De procedure. The questionnaire contained 12 items rated from 0 (disagree) to 3 (strongly agree). For evaluation purposes, we summarized the matching items and calculated a somatic complaints score, an awakeness score, and a hunger score. The common side effects observed in previous ATD studies, namely, headache, 
nausea, vomiting, dizziness, and sweating (48), were included in this questionnaire (the entire questionnaire is available in the Supplementary file). Participants completed this questionnaire every hour and immediately before and after the intake of the AA beverages.

Taste

For taste evaluation, an in-house questionnaire consisting of six items was employed (see Supplementary file). The consistency, the sulfuric taste, the difficulty of intake, and revulsion were assessed using a scale of 0 (disagree) to 3 (strongly agree) immediately after the intake of the AA beverages.

\section{Statistical analysis}

As primary analysis, the influence of the four different AA mixtures on TRP influx across the BBB was investigated using repeated measures analyses of variance (rmANOVAs) with treatment as the between-subjects factor, time as the within-subjects factor, and gender as a covariate in SPSS Version 21.0 for MacOS. The degrees of freedom for the ANOVA $F$-statistics were adjusted according to the Greenhouse-Geisser correction for nonsphericity $(p<0.05)$. Similar analyses were performed on TYR and PHE influx across the BBB, on [TRP]/[CAA] ratio, on tolerance (the somatic complaints score, awakeness score, and hunger score), and on the PA- and NAPANAS scores over time. In addition, separate ANOVAs, with the Greenhouse-Geisser correction, on the TRP influx for each group using time as a within-subjects factor were performed. One-way ANOVA was used to determine any significant differences in [TRP]/[CAA] ratio, in TRP, TYR, and PHE influx rates between the groups at baseline $\left(\mathrm{T}_{0}\right)$. The influences of the different side effects (e.g. headache, nausea, vomiting, dizziness, and sweating), part of the somatic complaints score, were assessed by rmANOVAs with time as the within-subjects factor and treatment as the between-subjects factor. One-way ANOVA with gender as a covariate was employed to analyze taste differences between the four mixtures. Gaussian distribution for all dependent variables was established with the Kolmogorov-Smirnov test for each of the four groups and for the groups formed by dividing the participants by gender. The level of statistical significance was set and kept at $p<0.05$. Because of the explorative nature of this study, alpha-adjustment was not employed. Post hoc power analyses were performed using $\mathrm{G}^{*}$ Power Version 3.1.9.2. for MacOS.

\section{Results}

All plasma AA concentrations of TRP, PHE, TYR, and BCAA are presented in Table 2. Table 3 includes all calculated influx values for TRP, TYR, PHE, (TYR + PHE), and BCAA across the BBB.

\section{TRP influx into the brain}

No significant differences in TRP influx were present at baseline $\left(\mathrm{T}_{0}\right)$ when comparing ATD, BAL, ATD PHE/LEU, $_{\text {, }}$ and $\mathrm{BAL}_{\mathrm{PHE} / \mathrm{LEU}}\left(F_{[3,71]}=2.005 ; p=n . s\right.$. $)$. An rmANOVA yielded main effects of time $\left(F_{[3.801,258.437]}=69.972\right.$; $p<0.001)$ and group $\left(F_{[3,68]}=97.577 ; p<0.001\right)$ and a significant time by group interaction $\left(F_{[11.402,258.437]}=\right.$ 25.875; $p<0.001)$. Bonferroni post hoc analyses indicated significant differences between each of the four groups (mean TRP influx BAL $>\mathrm{BAL}_{\mathrm{PHE} / \mathrm{LEU}}>\mathrm{ATD}>$ ATD $_{\text {PHE/LEU; }}$ see Supplementary Tables 2 and 3 for detailed results). The comparison of the influx curves for all groups is given in Fig. 1a. Separate rmANOVAs with time as the within-subjects factor indicated a main effect of time for each group. Table 4 summarizes the degrees of freedom (df) and $F$ - and $p$-values of these analyses. Time points of maximum TRP influx decline $\left(\mathrm{T}_{\max }\right)$, maximum decline in TRP influx in relation to baseline (Decline max $_{\text {) }}$, and overall TRP influx declines from baseline to time point $\mathrm{T}_{6}$ for each group are given in Table 5. Maximum decrease in TRP influx $4 \mathrm{~h}$ after intake was $11.9 \%$ higher for $\mathrm{ATD}_{\mathrm{PHE} / \mathrm{LEU}}$ compared with the decrease found for ATD. In contrast to ATD, $\mathrm{ATD}_{\mathrm{PHE} / \mathrm{LEU}}$, and $\mathrm{BAL} \mathrm{LHE}_{\mathrm{PHEU}}$ administration, a significant increase in TRP influx was detected in the BAL condition control group during the first $60 \mathrm{~min}$ after beverage administration $(p=0.002)$, while no significant changes in influx rates were detected over the entire period from $\mathrm{T}_{0}$ to $\mathrm{T}_{6}$. No effect of gender was detected $\left(F_{[1,67]}=2.537 ; p=\right.$ n.s. $)$. Post hoc power analysis showed that our study was adequately powered (power of 0.95 ) to detect effect sizes of 0.25 with an alpha level of 0.05 using a between-subjects repeated measures approach (ANOVA). In addition, we calculated Cohen's $d$-values as an estimate for effect sizes ( $d$-value of $d=0.24$ for the comparison of TRP-influx after ATD versus BAL administration; $d=0.14$ for the comparison of $\mathrm{ATD}_{\mathrm{PHE} / \mathrm{LEU}}$ and BAL $\left.\mathrm{PHE}_{\mathrm{PEU}}\right)$.

\section{TYR and PHE influx into the brain}

An rmANOVA on TYR influx rates indicated main effects of time $\left(F_{[2.122,144.270]}=226.885 ; p<0.001\right)$ and group $\left(F_{[3,68]}=11.191 ; p<0.001\right)$ and a significant time by group interaction $\left(F_{[6.365,144.270]}=2.255 ; p=0.038\right)$. Bonferroni post hoc analyses indicated that only ATD showed significant difference from the other three treatment conditions, while no significant difference could be found between the three of them. With regard to TYR influx, the curve progression for all treatment conditions was overall comparable with a strong decrease in TYR influx during the first hour (see Fig. 1b). While TYR influx after ATD administration was maximally decreased by $48 \%$ of the baseline value, influx after ATD $_{\text {PHE/LEU }}$ displayed a decrease of $71 \%$. Similar analyses for PHE influx values also showed main effects of time $\left(F_{[3.849}\right.$, $261.745]=204.575 ; p<0.001)$ and group $\left(F_{[3,68]}=77.348\right.$; 
Table 2. Blood concentrations of tryptophan, phenylalanine, tyrosine, and BCAA

\begin{tabular}{|c|c|c|c|c|c|c|c|c|}
\hline Parameter & Mixture & Baseline $\left(T_{0}\right)$ & $\mathrm{T}_{1}$ & $\mathrm{~T}_{2}$ & $\mathrm{~T}_{3}$ & $\mathrm{~T}_{4}$ & $T_{5}$ & $T_{6}$ \\
\hline \multirow[t]{4}{*}{ [TRP] in $\mu \mathrm{mol} / \mathrm{l}$} & ATD & $70 \pm 3$ & $117 \pm 22$ & $111 \pm 23$ & $95 \pm 22$ & $83 \pm 22$ & $75 \pm 23$ & $69 \pm 17$ \\
\hline & BAL & $68 \pm 3$ & $418 \pm 22$ & $437 \pm 23$ & $393 \pm 22$ & $372 \pm 22$ & $261 \pm 23$ & $178 \pm 17$ \\
\hline & ATD & $66 \pm 3$ & $107 \pm 22$ & $110 \pm 23$ & $111 \pm 22$ & $108 \pm 22$ & $105 \pm 23$ & $97 \pm 17$ \\
\hline & BAL & $68 \pm 3$ & $460 \pm 22$ & $407 \pm 23$ & $422 \pm 22$ & $409 \pm 22$ & $397 \pm 23$ & $308 \pm 17$ \\
\hline \multirow[t]{4}{*}{ [PHE] } & ATD & $51 \pm 24$ & $376 \pm 53$ & $484 \pm 92$ & $393 \pm 96$ & $419 \pm 117$ & $313 \pm 118$ & $242 \pm 115$ \\
\hline & BAL & $55 \pm 24$ & $329 \pm 53$ & $400 \pm 92$ & $330 \pm 96$ & $310 \pm 117$ & $284 \pm 118$ & $192 \pm 115$ \\
\hline & ATD & $112 \pm 24$ & $972 \pm 53$ & $1236 \pm 92$ & $1457 \pm 96$ & $1571 \pm 117$ & $1427 \pm 118$ & $1182 \pm 115$ \\
\hline & BAL & $86 \pm 24$ & $977 \pm 53$ & $1474 \pm 92$ & $1816 \pm 96$ & $1926 \pm 117$ & $1683 \pm 118$ & $1416 \pm 115$ \\
\hline \multirow[t]{4}{*}{ [TYR] } & ATD & $59 \pm 4$ & $108 \pm 8$ & $120 \pm 13$ & $109 \pm 14$ & $100 \pm 14$ & $94 \pm 15$ & $88 \pm 12$ \\
\hline & BAL & $55 \pm 4$ & $97 \pm 8$ & $97 \pm 13$ & $85 \pm 14$ & $79 \pm 14$ & $71 \pm 15$ & $60 \pm 12$ \\
\hline & $\mathrm{ATD}_{\mathrm{PHE} / \mathrm{LEU}}$ & $59 \pm 4$ & $132 \pm 8$ & $170 \pm 13$ & $193 \pm 14$ & $204 \pm 14$ & $197 \pm 15$ & $189 \pm 12$ \\
\hline & BAL & $62 \pm 4$ & $130 \pm 8$ & $169 \pm 13$ & $195 \pm 14$ & $207 \pm 14$ & $206 \pm 15$ & $196 \pm 12$ \\
\hline \multirow[t]{4}{*}[\mathrm{BCAA}]{} & ATD & $416 \pm 20$ & $1444 \pm 53$ & $1458 \pm 56$ & $|374 \pm 6|$ & $125 \mid \pm 57$ & $1040 \pm 64$ & $819 \pm 47$ \\
\hline & BAL & $412 \pm 20$ & $1278 \pm 53$ & $1297 \pm 56$ & $|249 \pm 6|$ & $1137 \pm 57$ & $905 \pm 64$ & $744 \pm 47$ \\
\hline & $\mathrm{ATD}_{\mathrm{PHE} / \mathrm{LEU}}$ & $405 \pm 20$ & $1078 \pm 53$ & $97 I \pm 56$ & $953 \pm 61$ & $822 \pm 57$ & $628 \pm 64$ & $485 \pm 47$ \\
\hline & BAL $L_{P H E / L E U}$ & $400 \pm 20$ & $1024 \pm 53$ & $945 \pm 56$ & $973 \pm 61$ & $827 \pm 57$ & $604 \pm 64$ & $446 \pm 47$ \\
\hline
\end{tabular}

Plasma amino acid concentrations (in $\mu \mathrm{mol} / \mathrm{l})$ of tryptophan, phenylalanine, tyrosine, and branched-chain amino acids (BCAA; including valine, leucine, and isoleucine) after the administration of four different amino acid mixtures: ATD (acute tryptophan depletion; challenge condition), BAL (control condition for ATD), ATD PHE/LEU (newly developed challenge condition), and BAL error are given for the different groups (each including 18 subjects) at the seven time points of blood withdrawal (T0-T6).

$p<0.001)$ and a significant time by group interaction $\left(F_{[11.548,261.745]}=2.255 ; p<0.001\right)$. Bonferroni post hoc analyses indicated significant differences between all groups, except of $\mathrm{ATD}_{\mathrm{PHE} / \mathrm{LEU}}$ versus $\mathrm{BAL}_{\mathrm{PHE} / \mathrm{LEU}}$ that did not present any significant results. PHE influx curves for all groups can be found in Fig. 1c. For ATD $_{\mathrm{PHE} / \mathrm{LEU}}$ and $\mathrm{BAL}_{\mathrm{PHE} / \mathrm{LEU}}$ PHE influx values showed an increase during the first $4 \mathrm{~h}$, followed by only a small decrease

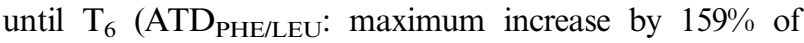

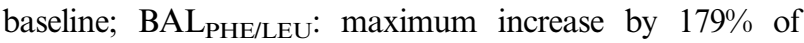

Table 3. Calculated influx values of particular amino acids across the BBB

\begin{tabular}{|c|c|c|c|c|c|c|c|c|}
\hline Parameter & Mixture & Baseline $\left(T_{0}\right)$ & $T_{1}$ & $\mathrm{~T}_{2}$ & $\mathrm{~T}_{3}$ & $\mathrm{~T}_{4}$ & $T_{5}$ & $T_{6}$ \\
\hline \multirow[t]{4}{*}{ TRP-influx } & ATD & $13.573 \pm 0.479$ & $6.912 \pm 0.603$ & $6.004 \pm 0.609$ & $5.715 \pm 0.645$ & $4.897 \pm 0.637$ & $5.485 \pm 0.752$ & $6.452 \pm 0.552$ \\
\hline & BAL & $13.313 \pm 0.479$ & $17.190 \pm 0.603$ & $16.533 \pm 0.609$ & $16.821 \pm 0.645$ & $16.864 \pm 0.637$ & $14.122 \pm 0.752$ & $14.236 \pm 0.552$ \\
\hline & ATD $_{\text {PHE/LEU }}$ & $12.135 \pm 0.479$ & $3.969 \pm 0.603$ & $3.434 \pm 0.609$ & $3.125 \pm 0.645$ & $2.934 \pm 0.637$ & $3.155 \pm 0.752$ & $3.557 \pm 0.552$ \\
\hline & BAL & $12.483 \pm 0.479$ & $12.449 \pm 0.603$ & $9.483 \pm 0.609$ & $8.828 \pm 0.645$ & $8.435 \pm 0.637$ & $8.939 \pm 0.752$ & $8.191 \pm 0.552$ \\
\hline \multirow[t]{4}{*}{ TYR-influx } & ATD & $5.647 \pm 0.297$ & $2.997 \pm 0.167$ & $3.002 \pm 0.173$ & $3.139 \pm 0.173$ & $2.911 \pm 0.189$ & $3.514 \pm 0.266$ & $4.078 \pm 0.311$ \\
\hline & BAL & $5.338 \pm 0.297$ & $2.054 \pm 0.167$ & $1.866 \pm 0.173$ & $1.877 \pm 0.173$ & $1.921 \pm 0.189$ & $1.959 \pm 0.266$ & $2.523 \pm 0.311$ \\
\hline & ATD $_{\text {PHE/LEU }}$ & $5.213 \pm 0.297$ & $1.905 \pm 0.167$ & $2.038 \pm 0.173$ & $1.964 \pm 0.173$ & $2.022 \pm 0.189$ & $2.285 \pm 0.266$ & $2.765 \pm 0.311$ \\
\hline & BAL $L_{P H E / L E U}$ & $5.464 \pm 0.297$ & $1.515 \pm 0.167$ & $1.530 \pm 0.173$ & $1.50 \mathrm{I} \pm 0.173$ & $1.582 \pm 0.189$ & $1.859 \pm 0.266$ & $2.348 \pm 0.311$ \\
\hline \multirow[t]{4}{*}{ PHE-influx } & ATD & $|0.39| \pm 0.630$ & $18.342 \pm 0.628$ & $19.733 \pm 0.918$ & $18.719 \pm 0.981$ & $19.493 \pm 1.120$ & $18.008 \pm 1.219$ & $16.975 \pm 1.119$ \\
\hline & BAL & $10.819 \pm 0.630$ & $14.706 \pm 0.628$ & $16.091 \pm 0.918$ & $14.764 \pm 0.98 \mid$ & $14.540 \pm 1.120$ & $14.522 \pm 1.219$ & $14.088 \pm 1.119$ \\
\hline & ATD $_{\text {PHE/LEU }}$ & $12.234 \pm 0.630$ & $26.229 \pm 0.628$ & $28.66 \mathrm{I} \pm 0.918$ & $30.627 \pm 0.98 I$ & $31.642 \pm 1.120$ & $30.493 \pm 1.219$ & $28.378 \pm 1.119$ \\
\hline & BAL & $11.978 \pm 0.630$ & $24.250 \pm 0.628$ & $29.411 \pm 0.918$ & $32.387 \pm 0.98 \mathrm{I}$ & $33.428 \pm 1.120$ & $31.321 \pm 1.219$ & $29.325 \pm 1.119$ \\
\hline \multirow[t]{4}{*}{ BCAA-influx } & ATD & $11.968 \pm 0.453$ & $15.582 \pm 0.448$ & $|5.1| 2 \pm 0.50 \mid$ & $15.502 \pm 0.570$ & $14.340 \pm 0.544$ & $14.018 \pm 0.612$ & $12.901 \pm 0.435$ \\
\hline & BAL & $11.736 \pm 0.453$ & $12.198 \pm 0.448$ & $\mathrm{II} .692 \pm 0.50 \mathrm{I}$ & $12.092 \pm 0.570$ & $11.570 \pm 0.544$ & $10.245 \pm 0.612$ & $10.809 \pm 0.435$ \\
\hline & ATD $_{\text {PHE } / L E U}$ & $10.937 \pm 0.453$ & $8.505 \pm 0.448$ & $7.131 \pm 0.501$ & $6.524 \pm 0.570$ & $5.587 \pm 0.544$ & $4.464 \pm 0.612$ & $3.872 \pm 0.435$ \\
\hline & BAL & $10.814 \pm 0.453$ & $7.352 \pm 0.448$ & $6.171 \pm 0.501$ & $5.968 \pm 0.570$ & $5.044 \pm 0.544$ & $3.958 \pm 0.612$ & $3.164 \pm 0.435$ \\
\hline
\end{tabular}

Calculated influx values (in $\mathrm{nmol} / \mathrm{min} / \mathrm{g}$ brain tissue) for tryptophan (TRP), tyrosine (TYR), phenylalanine (PHE), and branched-chain amino acids (BCAA; including valine, leucine, and isoleucine) across the blood-brain barrier after the administration of four different amino acid mixtures: ATD (acute tryptophan depletion; challenge condition), BAL (control condition for ATD), ATD PHE/LEU (newly developed challenge condition), and BALPHE/ LEU (control condition for ATD PHE/LEU). Mean values \pm standard error are presented for the different groups (each including 18 subjects) at the seven time points of blood withdrawal $\left(T_{0}-T_{6}\right)$. 
(a)

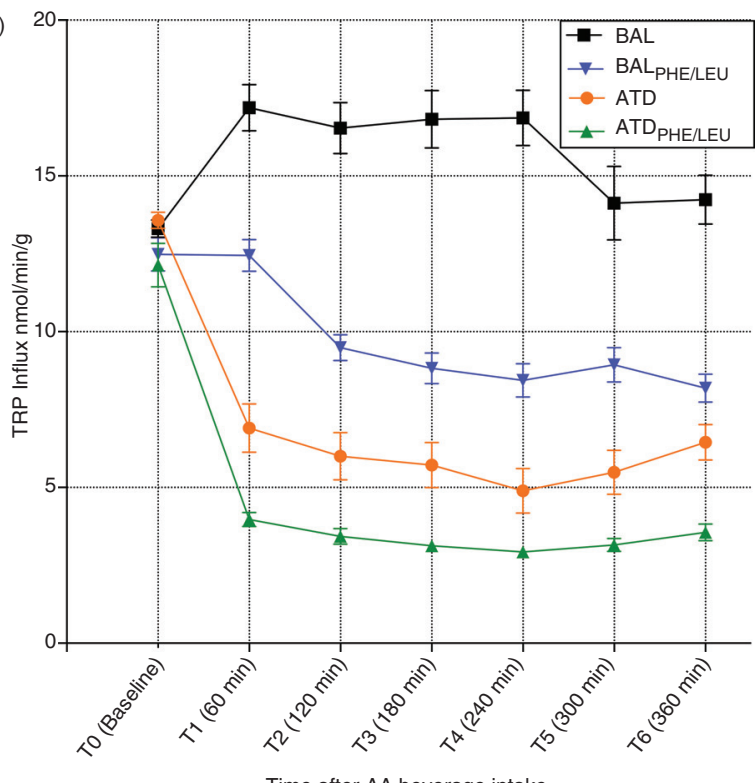

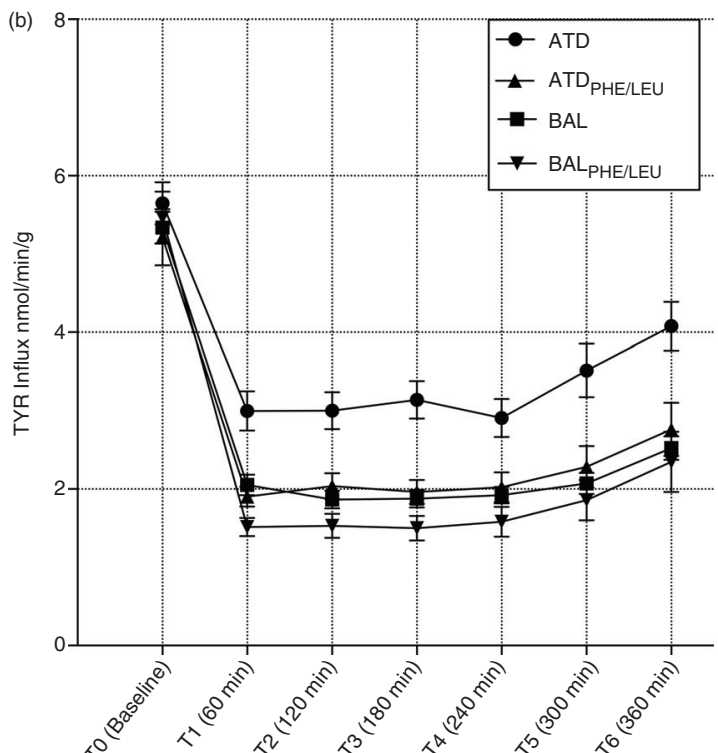

Time after AA beverage intake

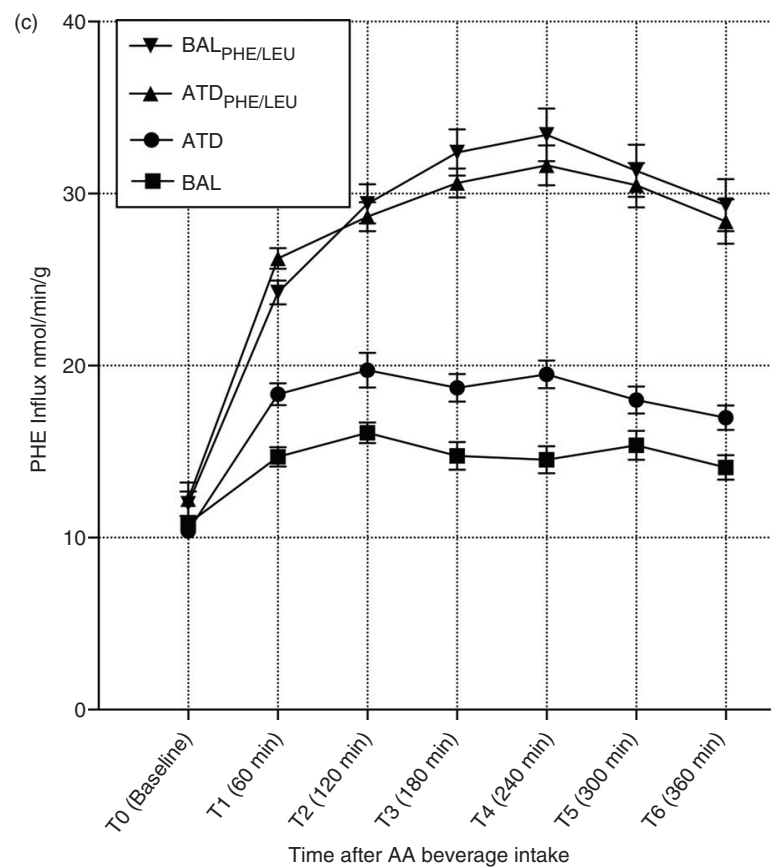

Fig. 1. (a, b, c) Influx curves (nmol/min/g brain tissue) of tryptophan (TRP), tyrosine, and phenylalanine across the blood-brain barrier at baseline (T0) and time points T1-T6 after the intake of the ATD Moja-De amino acid (AA) mixture, its balanced control condition (BAL), the newly developed tryptophan depletion protocol $\mathrm{ATD}_{\mathrm{PHE} / \mathrm{LEU}}$, and the corresponding control condition (BAL $\left.\mathrm{PHE}_{\mathrm{LEU}}\right)$. Data are given as the mean values, with bars representing the standard errors.

baseline). In comparison, a much smaller increase of PHE influx appeared after ATD (maximum increase by $89 \%$ of baseline) and BAL (increase by $49 \%$ of baseline) administration.

\section{$[T R P] /[C A A]$}

Figure 2 shows the time course of [TRP]/[CAA] ratios for the four treatment conditions, which offers a comparable development with regard to TRP influx calculations, but with a focus on plasma concentrations. No significant differences in the ratios were present at baseline $\left(T_{0}\right)$ when comparing ATD, BAL, ATD PHE/LEU, and BAL $_{\text {PHE/LEU }}$ $\left(F_{[3,71]}=0.898 ; p=\right.$ n.s. $)$. An rmANOVA yielded main effects of time $\left(F_{[4.213,286.489]}=5.447 ; p<0.001\right)$ and group $\left(F_{[3,68]}=73.368 ; p<0.001\right)$ and a significant time by group interaction $\left(F_{[12.639,286.489]}=17.960 ; p<0.001\right)$. Bonferroni post hoc analyses indicated significant differences between each of the four groups except of no significant result for 
Table 4. Results of rmANOVA on tryptophan influx values

\begin{tabular}{lcccc}
\hline & ATD & BAL & ATD $_{\text {PHE/LEU }}$ & BAL $L_{P H E / L E U}$ \\
\hline $\mathrm{df}$ & $2.130,36.206$ & $3.136,53.314$ & $1.269,21.579$ & $4.057,68.962$ \\
$\mathrm{~F}$ & 86.110 & 7.646 & 120.067 & 20.645 \\
$P$ & $<0.001$ & $<0.001$ & $<0.001$ & $<0.001$ \\
\hline
\end{tabular}

Results of separate repeated measurements analyses of variance (rmANOVAs) on tryptophan (TRP) influx across the blood-brain barrier for ATD (challenge condition), BAL (control condition for

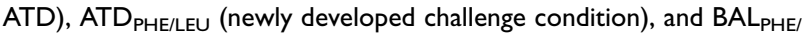
LEU (control condition for ATD PHE/LEU), with time as the within-subjects factor. df, degrees of freedom; $F, F$ values; $p$, statistical significance.

ATD and $\mathrm{ATD}_{\mathrm{PHE} / \mathrm{LEU}}$. Maximum decrease in [TRP]/[CAA] ratio is reached $4 \mathrm{~h}$ after intake $\left(\mathrm{T}_{4}\right)$ for both protocols. ATD and $\mathrm{ATD}_{\mathrm{PHE} / \mathrm{LEU}}$ reduced the ratio by 66 and $65 \%$, respectively, when compared with baseline. Values of all calculated ratios are given in Table 6, and additional graphs for $[\mathrm{TYR}] /[\mathrm{CAA}]$ and $[\mathrm{PHE}] /[\mathrm{CAA}]$ ratios of all groups are presented in Supplementary Fig. S1a and S1b.

\section{Taste}

One-way ANOVA of the taste evaluation scores with gender as a covariate yielded no significant difference in taste perceptions between the groups $\left(F_{[3,67]}=0.997\right.$; $p=$ n.s. $)$ and no effect of gender $\left(F_{[1,67]}=0.102 ; p=\right.$ n.s. $)$.

\section{Challenge tolerance}

An rmANOVA evaluation of challenge tolerance indicated a main effect of time for the analysis of the hunger score $\left(F_{[4.760,314.190]}=4.457 ; p=0.001\right)$; post hoc testing indicated a significant increase in hunger from baseline $\left(\mathrm{T}_{0}\right)$ to $\mathrm{T}_{6}(p=0.001)$. Similar analyses of the awakeness score also yielded a main effect of time $\left(F_{[4.182,275.991]}=9.741\right.$; $p<0.001)$. Within the $6 \mathrm{~h}$ from baseline $\left(\mathrm{T}_{0}\right)$ to $\mathrm{T}_{6}$, the awakeness score decreased significantly $(p=0.001)$, but participants felt most tired at $\mathrm{T}_{2}$. The main effects of time $\left(F_{[4.018,265.208]}=5.077 ; p=0.001\right)$ and group $\left(F_{[3,66]}=3.939 ; p=0.012\right)$ were detected for the somatic complaints score and indicated an increase in somatic

Table 5. Descriptive data of tryptophan depletion graphs

\begin{tabular}{lcccc}
\hline & ATD & BAL & ATD $_{\text {PHE/LEU }}$ & BAL \\
\hline$T_{\max / \text { (mEU }}(\min )$ & 240 & 60 & 240 & 360 \\
Decline $_{\max }(\%)$ & 63.9 & 29.1 (increase) & 75.8 & 34.4 \\
Decline $_{\text {T0-T6 }}(\%)$ & 52.5 & 6.9 (increase) & 70.7 & 34.4 \\
\hline
\end{tabular}

Time points of maximum tryptophan (TRP) influx decline $\left(T_{\text {max }}\right)$, maximum declines of TRP influx in relation to baseline $\left(T_{0}\right)\left(\right.$ Decline $\left._{\max }\right)$, and overall TRP influx declines from baseline $\left(T_{0}\right)$ to time point $T_{6}$ (time point of last blood sample) for the groups ATD (challenge condition), BAL (control condition for ATD), ATD PHELLEU (newly developed challenge condition), and BAL PHE/LEU (control condition for ATD PHE/LEU). complaints over time. Bonferroni post hoc analyses showed a significant difference between $B \mathrm{BL}_{\mathrm{PHE} / \mathrm{LEU}}$ and BAL administration $(p=0.012)$. The average somatic complaints score related to $\mathrm{BAL}_{\mathrm{PHE} / \mathrm{LEU}}$ administration (average score $0.708 \pm 0.117$ ) was four times greater than the values reported by the BAL condition group (average score $0.169 \pm 0.121)$. While the participants overall felt more unwell $2 \mathrm{~h}\left(\mathrm{~T}_{2}\right)$ after the intake of the AA mixtures $(p=0.022)$, the somatic complaints scores decreased from $\mathrm{T}_{2}$ to $\mathrm{T}_{4}(p=0.002)$, with only a slight, non-significant increase observed at $\mathrm{T}_{6}$ compared with the baseline score. At baseline, there were no significant differences in somatic complaints scores between the groups $\left(F_{[3,68]}=0.169 ; p=\right.$ n.s. $)$. Analyses of each individual item in the somatic complaints score showed a comparable development of nausea. The report of nausea significantly increased from $\mathrm{T}_{0}$ to $\mathrm{T}_{2}(p=0.046)$ and then significantly decreased from $\mathrm{T}_{2}$ to $\mathrm{T}_{4}(p=0.014)$. No gender effects were detected in the hunger $\left(F_{[1,65]}=\right.$ $0.192 ; p=$ n.s. $)$, awakeness $\left(F_{[1,65]}=0.769 ; p=\right.$ n.s. $)$, or somatic complaints scores $\left(F_{[1,65]}=0.179 ; p=\right.$ n.s. $)$.

\section{Mood assessment (PANAS)}

An rmANOVA of the PA score showed a main effect of time $\left(F_{[3.335,220.091]}=54.110 ; p<0.001\right)$, but no significant effect of group $\left(F_{[3,65]}=0.215 ; p=\right.$ n.s. $)$ or gender $\left(F_{[1,} 65\right]=0.933 ; p=$ n.s. $)$. Compared with the baseline score, a decrease in the PA score of $28.5 \%$ at $\mathrm{T}_{6}$ was observed $(p<0.001)$. The NA score also decreased $\left(11.4 \%\right.$ from baseline to $\mathrm{T} 6 ; F_{[3.198,211.086]}=$ $12.502 ; p<0.001$, rmANOVA) regardless of gender $\left(F_{[1,65]}=0.268 ; p=\right.$ n.s. $)$.

\section{Discussion}

The results of this pilot study indicate that dietary administration of ATD $_{\text {PHE/LEU }}$ led to a greater depletion magnitude with regard to the TRP influx across the BBB compared with the depletion observed when using the ATD Moja-De protocol, but not with regard to plasma TRP concentrations because of an unintended initial short increase in plasma TRP concentrations that was observed after $\mathrm{ATD}_{\mathrm{PHE} / \mathrm{LEU}}$ intake (see further below in this section for a discussion). The found effect on TRP influx across the BBB was observed even though

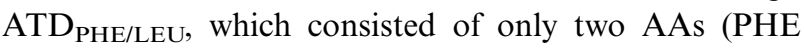
and LEU), was administered in a lower overall AA dose. Participants evaluated the tolerance level of the new AA challenge beverage as acceptable. No situations or events necessitating premature termination of the study occurred (such as participants vomiting during measurements or other incidents affecting ratings). Thus, several criteria for a potentially valid and effective ATD-test protocol were already achieved, while other aspects such as the observed unexpected increase in plasma TRP levels 


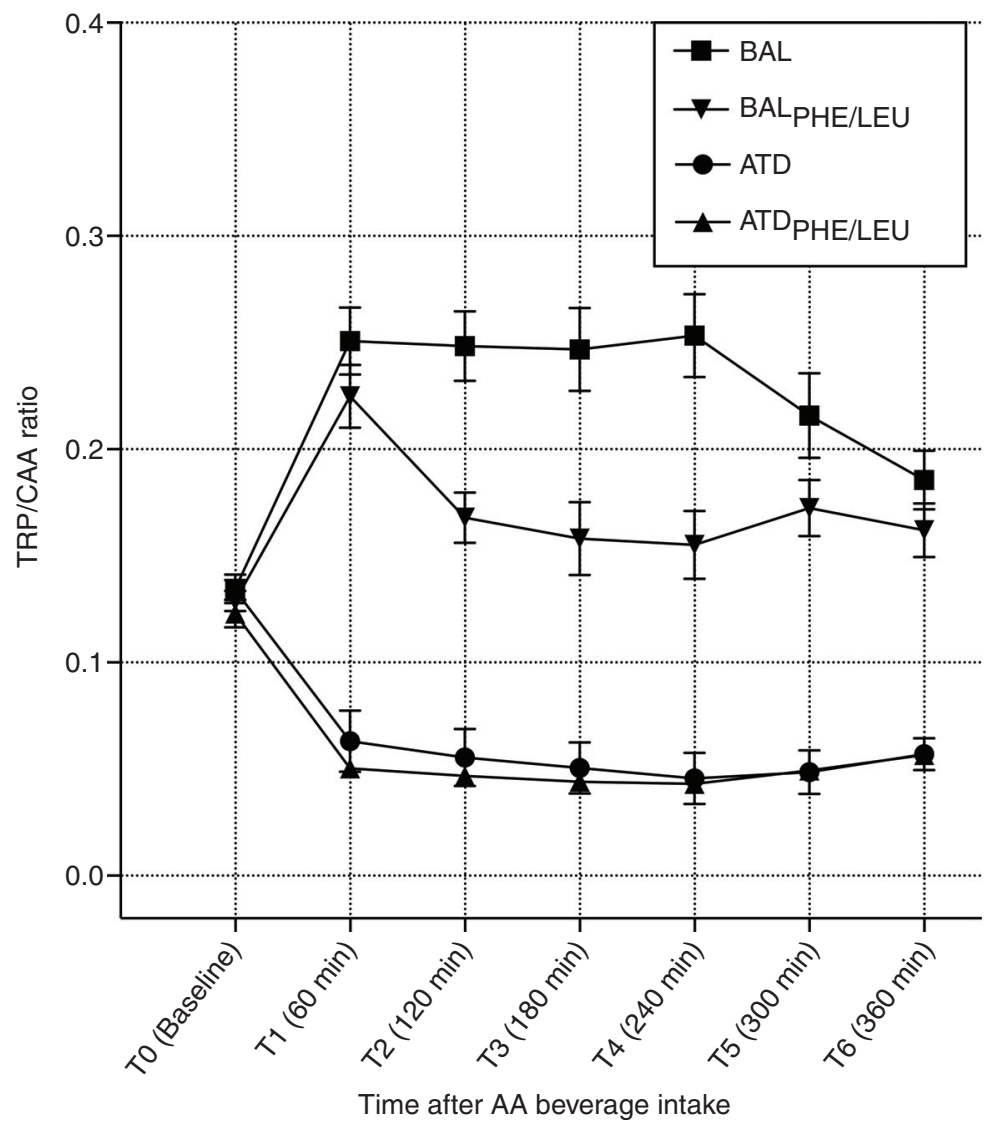

Fig. 2. Curve progression for calculated TRP/CAA ratios from baseline (T0) to time point T6 after the intake of the ATD MojaDe amino acid (AA) mixture, its balanced control condition (BAL), the newly developed tryptophan depletion protocol $\mathrm{ATD}_{\mathrm{PHE} / \mathrm{LEU}}$, and the corresponding control condition (BAL $\left.\mathrm{PHE}_{\mathrm{LEU}}\right)$. Data are given as the mean values, with bars representing the standard errors.

require further improvements and future studies in order to validate the findings of the current pilot study.

The altered composition of $\mathrm{ATD}_{\mathrm{PHE} / \mathrm{LEU}}$, including only two AAs, in contrast to the ATD Moja-De that includes seven different AAs, should lead to a greatly simplified production of the AA beverage. Moreover, it also enables facilitated monitoring of relevant blood parameters (i.e. AA concentrations) as their number was consequently reduced. Such monitoring was further simplified by the development and use of ELISA and BCAA test kits (Immundiagnostik AG, Bensheim, Germany). ATD $_{\text {PHE/LEU }}$ uses the same body weight-adapted dosing regimen of the ATD AA mixtures that was first successfully applied in the ATD Moja-De protocol $(36,37)$. This well-tolerated dosing regimen is based on the assumption of a positive correlation between body weight and plasma TRP levels $(36,37)$ and, as a significant advantage, leads to a possible application for children and adolescents with fewer side effects $(42-44,49,50)$.

The greater effectiveness of ATD $\mathrm{PHE}_{\mathrm{LEU}}$ in terms of a greater reduction in TRP influx across the BBB when compared with ATD Moja-De could be seen as an advantage, whereas the found effects on plasma TRP concentrations require further investigation. The reason for the detected effects on TRP influx into the brain may lie in the different affinities of the LNAAs to the common $\mathrm{L}-1$ transporter. The Michaelis constant $\left(\mathrm{K}_{\mathrm{M}}\right)$ represents a measurement of these particular affinities. Precisely, it is indicative of the specific substrate concentration needed to achieve the half-maximal rate of turnover of an enzyme. Thus, a lower $K_{M}$ value of a substrate is associated with its higher affinity to the related enzyme. Smith et al. (16) determined the values of transport constants for seven LNAAs, including PHE $\left(0.011 \mu \mathrm{mol} \cdot \mathrm{ml}^{-1}\right)$ and LEU $\left(0.029 \mu \mathrm{mol} \cdot \mathrm{ml}^{-1}\right)$, in anesthetized rats. It must be acknowledged that transferring findings from rodent research to humans is subject to limitations. However, these values for the respective transport constants can serve as a first rough estimate, and the high and significant positive correlations between TRP influx and the TRP/CAA ratio (a previous measure for the effects of ATD) are consistent with the theoretical considerations for using MichaelisMenten kinetics to characterize TRP influx across the BBB. The high affinity of PHE and LEU to L-1, compared 
Table 6. Amino acid ratios

\begin{tabular}{|c|c|c|c|c|c|c|c|c|}
\hline Parameter & Mixture & Baseline $\left(T_{0}\right)$ & $T_{1}$ & $\mathrm{~T}_{2}$ & $\mathrm{~T}_{3}$ & $\mathrm{~T}_{4}$ & $T_{5}$ & $T_{6}$ \\
\hline \multirow[t]{4}{*}[\mathrm{TRP}]{$/[\mathrm{CAA}]$} & ATD & $0.134 \pm 0.006$ & $0.063 \pm 0.013$ & $0.055 \pm 0.012$ & $0.051 \pm 0.014$ & $0.046 \pm 0.014$ & $0.049 \pm 0.014$ & $0.057 \pm 0.010$ \\
\hline & BAL & $0.135 \pm 0.006$ & $0.251 \pm 0.013$ & $0.248 \pm 0.012$ & $0.247 \pm 0.014$ & $0.253 \pm 0.014$ & $0.204 \pm 0.014$ & $0.186 \pm 0.010$ \\
\hline & ATD ${ }_{\text {PHE/LEU }}$ & $0.123 \pm 0.006$ & $0.050 \pm 0.013$ & $0.047 \pm 0.012$ & $0.044 \pm 0.014$ & $0.043 \pm 0.014$ & $0.0493 \pm 0.014$ & $0.057 \pm 0.010$ \\
\hline & BAL & $0.129 \pm 0.006$ & $0.225 \pm 0.013$ & $0.168 \pm 0.012$ & $0.158 \pm 0.014$ & $0.155 \pm 0.014$ & $0.172 \pm 0.014$ & $0.162 \pm 0.010$ \\
\hline \multirow[t]{4}{*}[\mathrm{PHE}]{$/[\mathrm{CAA}]$} & ATD & $0.094 \pm 0.043$ & $0.232 \pm 0.037$ & $0.320 \pm 0.078$ & $0.293 \pm 0.077$ & $0.329 \pm 0.087$ & $0.308 \pm 0.117$ & $0.254 \pm 0.139$ \\
\hline & BAL & $0.102 \pm 0.043$ & $0.182 \pm 0.037$ & $0.220 \pm 0.078$ & $0.193 \pm 0.077$ & $0.193 \pm 0.087$ & $0.213 \pm 0.117$ & $0.191 \pm 0.139$ \\
\hline & ATD ${ }_{\text {PHE/LEU }}$ & $0.206 \pm 0.043$ & $0.759 \pm 0.037$ & $1.039 \pm 0.078$ & $1.183 \pm 0.077$ & $1.399 \pm 0.087$ & $1.548 \pm 0.117$ & $1.561 \pm 0.139$ \\
\hline & BAL $L_{P H E / L E U}$ & $0.163 \pm 0.043$ & $0.610 \pm 0.037$ & $0.952 \pm 0.078$ & $1.155 \pm 0.077$ & $1.334 \pm 0.087$ & $1.403 \pm 0.117$ & $1.462 \pm 0.139$ \\
\hline \multirow[t]{4}{*}[\mathrm{TYR}]{$/[\mathrm{CAA}]$} & ATD & $0.108 \pm 0.006$ & $0.056 \pm 0.004$ & $0.059 \pm 0.004$ & $0.059 \pm 0.005$ & $0.059 \pm 0.006$ & $0.068 \pm 0.009$ & $0.081 \pm 0.012$ \\
\hline & BAL & $0.103 \pm 0.006$ & $0.048 \pm 0.004$ & $0.046 \pm 0.004$ & $0.043 \pm 0.005$ & $0.045 \pm 0.006$ & $0.047 \pm 0.009$ & $0.056 \pm 0.012$ \\
\hline & ATD $D_{\text {PHE/LEU }}$ & $0.105 \pm 0.006$ & $0.063 \pm 0.004$ & $0.075 \pm 0.004$ & $0.079 \pm 0.005$ & $0.086 \pm 0.006$ & $0.103 \pm 0.009$ & $0.124 \pm 0.012$ \\
\hline & BAL $L_{P H E / L E U}$ & $0.112 \pm 0.006$ & $0.054 \pm 0.004$ & $0.062 \pm 0.004$ & $0.063 \pm 0.005$ & $0.070 \pm 0.006$ & $0.085 \pm 0.009$ & $0.112 \pm 0.012$ \\
\hline [PHE + TYR]/ & ATD & $0.226 \pm 0.051$ & $0.318 \pm 0.043$ & $0.428 \pm 0.093$ & $0.405 \pm 0.093$ & $0.447 \pm 0.103$ & $0.451 \pm 0.145$ & $0.395 \pm 0.194$ \\
\hline \multirow[t]{3}{*}[\mathrm{BCAA}+\mathrm{TRP}]{} & BAL & $0.229 \pm 0.051$ & $0.250 \pm 0.043$ & $0.289 \pm 0.093$ & $0.255 \pm 0.093$ & $0.257 \pm 0.103$ & $0.285 \pm 0.145$ & $0.270 \pm 0.194$ \\
\hline & ATD $_{\text {PHE/LEU }}$ & $0.359 \pm 0.051$ & $0.963 \pm 0.043$ & $1.373 \pm 0.093$ & $1.594 \pm 0.093$ & $1.927 \pm 0.103$ & $2.268 \pm 0.145$ & $2.450 \pm 0.194$ \\
\hline & BAL & $0.3|3 \pm 0.05|$ & $0.756 \pm 0.043$ & $1.188 \pm 0.093$ & $1.452 \pm 0.093$ & $1.723 \pm 0.103$ & $1.882 \pm 0.145$ & $2.096 \pm 0.194$ \\
\hline
\end{tabular}

Ratios of tryptophan, phenylalanine, tyrosine, and branched-chain amino acids (BCAA; including valine, leucine, and isoleucine) and their competing amino acids (CAA) after the administration of four different amino acid mixtures: ATD (acute tryptophan depletion; challenge condition), BAL (control

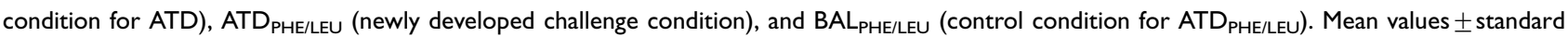
error are given for the different groups (each including 18 subjects) at the seven time points of blood withdrawal (T0-T6).

with all other LNAAs, may have caused the best displacement of TRP at the common transporter and therefore was possibly associated with a greater reduction of TRP influx across the BBB. By contrast, the ATD Moja-De, containing a mixture of seven different AAs with partly lower $\mathrm{K}_{M}$ values, was less effective with regard to the competition of the AAs against TRP, resulting in a smaller TRP depletion magnitude in terms of TRP influx across the BBB compared with the $\mathrm{ATD}_{\mathrm{PHE} / \mathrm{LEU}}$ depletion magnitude. It is possible that the use of this competitive mechanism in the respective AAs enables the potential effectiveness of TRP depletion after ATD PHE/LEU administration, despite the simultaneous reduction in the overall AA dose and thus a possibly decreased stimulation of protein synthesis in the liver when compared to other ATD protocols. This could have impacted the observed findings with regard to changes in plasma TRP concentrations and a possible redistribution between free and protein-bound TRP. The observed TRP influx curves of the old and new challenge protocols over the entire study duration resulted in maximum depletion in terms of decreased TRP influx across the BBB at $4 \mathrm{~h}$ after intake for both challenge procedures. In particular, TRP influx correlated highly and significantly with the TRP/CAA ratio for both ATD challenge conditions used (see Supplementary Tables S5 and S6 and Fig. S2a and S2b), but the influx rates are likely to be a more adequate reflection of TRP availability in the brain as they also take the individual affinities of the different amino acids to the L-1 transport system at the BBB into account.
It is important to keep in mind that this work only claims to be a first pilot study. Therefore, the assessment of brain TRP and 5-HT concentrations after ATD PHE/LEU $_{\text {applica- }}$ tion in rodents has to be an essential part of future research as well as PET-studies and the measurement of 5-hydroxyindoleacetic acid (5-HIAA) concentrations (the primary metabolite of 5-HT) in human cerebrospinal fluid in order to validate the effectiveness of ATD $_{\text {PHE/LEU }}$ revealed by this study. A recent study by Sánchez et al. (12) has provided preliminary evidence for the effectiveness of simplified ATD protocols using a three-AA-formulation (PHE, LEU, and ILE); however, future studies in humans assessing cerebrospinal fluid (CSF) 5-HIAA concentrations after intake of the challenge procedure are needed.

The absence of any gender effects in the blood parameters was an expected outcome. Although central nervous 5-HT synthesis is known to significantly differ in males and females (51), we monitored peripheral TRP and LNAA plasma levels for TRP influx calculations; these parameters are not influenced by gender-specific alterations in TRP influx and related central nervous 5-HT synthesis. The performed power analyses confirmed that this study was adequately powered to detect small effects, and Cohen's d calculations indicated small estimates for effect sizes for the analyses conducted.

Specificity for depleting central nervous 5-HT synthesis (in particular with regard to possible effects on dopamine and other neurotransmitters) is always an important point to be considered with regard to all available ATD-related amino acid formulations. Previous research from our 
group indicated that the Moja-De protocol does not affect central nervous dopamine synthesis in mice (38). As such evidence is currently missing for the newly developed protocol ATD PHE/LEU, we looked at TYR and PHE plasma values and their calculated influx into the brain. The strong decrease in TYR influx after challenge application, as described above, questions the specificity of the newly developed ATD PHE/LEU condition. The phenomenon of highly increasing PHE influx values in the $\mathrm{ATD}_{\mathrm{PHE} / \mathrm{LEU}}$ protocol group can be explained by its high PHE concentrations. As the strong decrease in TYR influx indicates, the high PHE dose does not mitigate the decrease in TYR influx (calculated from peripheral blood values). Whether the high PHE amount in the $\mathrm{ATD}_{\mathrm{PHE} / \mathrm{LEU}}$ mixture directly affects central nervous concentrations of TYR formation can only be answered using future neurochemical challenge studies. However, specificity for respective neurotransmitters is always a critical issue when dealing with different TRP depletion protocols. This is a general problem in ATD-related research in humans and affects all currently available ATD amino acid formulations. Badawy et al. (52) provided an approach to enhance ATD formulations' specificity by decreasing the amount of BCAAs. In accordance with this, the reduction of the LEU amount in our newly developed $\mathrm{ATD}_{\mathrm{PHE} / \mathrm{LEU}}$ protocol might be a potential avenue for future research. An unintended rather substantial increase in plasma TRP was observed after ATD $_{\text {PHE/LEU }}$ intake besides the fact that no TRP was ingested, and future studies are needed to investigate and replicate this unknown effect. Protein synthesis can be stimulated by the ingested amino acids, which in turn might have led to a possible redistribution between free and protein-bound TRP. Following this train of thought, the amount of free TRP possibly was slightly higher while the overall balance remained unaffected. It also needs to be outlined that TRP-related metabolites, such as quinolinic acid, kynurenic acid, and tryptamine that can be changed due to administration of the balanced mixture, can influence brain function. This stands in line with the issue of the specificity of all ATD/BAL paradigms currently available. Finally, the reason why the fasting TRP levels ( $\mathrm{T} 0$ ) were higher than those mentioned in the literature is not clear but worth noting and might be specific to the investigated sample.

Another issue regarding our new ATD challenge protocol is the absence of an appropriate control condition that 'maintains baseline values without altering the biochemical or behavioral parameters' (52). The observed rise in TRP influx approximately $1 \mathrm{~h}$ after administration of the BAL control condition seems to be a logical consequence of the enhanced TRP availability because the BAL mixture contained TRP. For this particular reason, the decline in TRP influx of $34.4 \%$ after BAL PHE/LEU $_{\text {admin- }}$ istration over the 6-h period appears to be counterintuitive.
When comparing $B A L_{P H E / L E U}$ with its challenge condition $\mathrm{ATD}_{\mathrm{PHE} / \mathrm{LEU}}$, the administration of $\mathrm{BAL}_{\mathrm{PHE} / \mathrm{LEU}}$ led to a depletion of approximately $50 \%$ in magnitude of the depletion observed following ATD $_{\text {PHE/LEU adminis- }}$ tration. The large amount of PHE in the $\mathrm{BAL}_{\mathrm{PHE} / \mathrm{LEU}}$ mixture may be a possible explanation for this particular phenomenon, which in turn could lead to an unexpected increase in displacement of TRP at the BBB in this control condition. To address this particular problem, either an increase in TRP or decreased levels of PHE or LEU in the control condition could be evaluated in future research in order to establish a valid control condition for ATD $\mathrm{AHE}_{\mathrm{PHEU}}$.

Both protocols that were applied in this study, ATD and BAL on the one hand and $\mathrm{ATD}_{\mathrm{PHE} / \mathrm{LEU}}$ and BAL $\mathrm{PHE}_{\mathrm{PHEU}}$ on the other hand, were well tolerated by the subjects. In particular, we did not observe any headaches, vomiting, dizziness, or sweating as indicated by the itemizing analyses of our somatic complaints score (see Supplementary Table S3). There were no dropouts caused by side effects. Hence, this requirement of a valid test protocol was met. Although we could not observe a significant group effect for the somatic complaints score between ATD

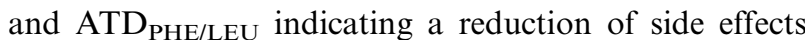
in the new challenge condition, we achieved the goal of maintaining the high-grade safety of the established ATD Moja-De.

The monitored increase in hunger throughout the study day most likely resulted from the 6-h abstinence from food and a breakfast overall low in nutrition. The lack of any group effect in hunger ratings supports the previous assumption. This observation justifies the necessity of the low-protein breakfast at the beginning of the study day. However, although its carbohydrate content can, to some extent, affect plasma TRP concentration and binding and levels of BCAA, it would not be justifiable to demand fasting beginning at 8 p.m. the night before the study day till 3 p.m. on the study day. Furthermore, the collection of additional behavioral, cognitive, and emotional data during this study for future analysis would have been likely to be skewed by significant fasting effects if no breakfast had been given. In this context, a variety of ATD-using studies also administer low-protein meals during the depletion procedure (53-56). In the present sample, we attribute the occurrence of increasing tiredness during the study day to external circumstances, such as the study environment and the long testing period, as opposed to a side effect of the protocol. The missing group effect can be seen as somewhat indicative for this phenomenon. Moreover, breakfast intake could also have impacted side effect ratings as assessed within the present study.

As stated above, we found an effect of group and time for the somatic complaints score. In the separated item analyses, nausea is the only side effect showing a main effect of time and displays an equal development. 
Although few ATD studies focused on the examination of side effects, nausea appears to be a frequent problem in these particular studies $(31,48)$. It is very likely that the ingestion of a substantial amount of crystalline AAs (Moja-De: $6.5 \mathrm{~g} / 10 \mathrm{~kg}$ body weight, ATD $_{\text {PHE/LEU }}$ $6.2 \mathrm{~g} / 10 \mathrm{~kg}$ body weight) that remain in the stomach until dissolution in the acidic environment contributed to the reports of nausea (57). The missing group effect in somatic complaint investigations partially supports this assumption because all groups were equally affected. Moreover, post hoc analyses of the somatic complaints scores revealed a significant difference between BAL

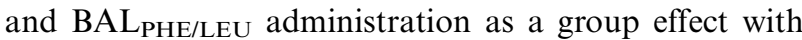
an average somatic complaints score four times greater after $\mathrm{BAL}_{\mathrm{PHE} / \mathrm{LEU}}$ intake. However, with a maximal somatic complaints score of 24 and a minimal score of 0 , the very low mean value observed in this study was not indicative of the intolerability of the new control condition. A modification of the BAL $\mathrm{L}_{\mathrm{PHE} / \mathrm{LEU}}$ control condition, as mentioned above, might help improve the side effect profile of BAL $\mathrm{PHE/LEU}$.

Presently, there is no ATD-specific validated questionnaire available for somatic complaint or taste assessment. This study provides first questionnaire-based pilot data and certainly requests validation in future ATD studies. In particular, further refinement is needed regarding the gradual and sensible detection of symptom nuances.

Both positive (PA) and negative (NA) PANAS assessments decreased over the 6-h study duration. In addition to these controversial findings, no group effect was detected, which suggests that the ratings were influenced by the situation during measurements and, perhaps, not a direct consequence of altered brain 5-HT function. This is in agreement with previous findings which suggested that ATD's influence on mood was largely dependent on the susceptibility of the study sample, and this variability led to a lack of clear mood effects in many cases $(58,59)$. However, the assessment of mood only served as an ancillary controlling variable in this study, and these results do not significantly impact or question its major findings.

Although subjects took the amino acid mixtures without any major problems and the new mixture was well tolerated in our study, the uncomfortable palatability seen in all groups certainly requests further modification to ensure trouble-free utilization. Bitterness in general is a problematic issue in all ATD studies as it arises from the specific biochemical structure of polar and hydrophobic groups in some amino acids (60). The reduction of the overall amount and dose as well as the absence of the worst tasting AAs (i.e. MET, cysteine, and arginine) might have contributed to an easier administration of $\mathrm{ATD}_{\mathrm{PHE} / \mathrm{LEU}}$; however, improvements are still required.
For this particular purpose, we suggest to add some of the conventional flavoring supplements, such as vanilla, peppermint, raspberry, and lemonade, with low sugar substitutes to our challenge procedure.

The between-subjects design is a limitation of this study. Behavioral paradigms conducted as a part of this study (the findings will be the subject of further publications) did not allow for a within-subject repeated measures design due to possible learning effects; a withinsubjects design could be used in further investigations.

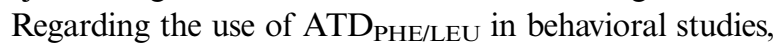
the high amount of LEU might present a problem, in particular as LEU is known to alter glutamate formation within the leucine-glutamate cycle $(61,62)$. Since LEU might also affect various other parameters, a separate analysis of LEU blood values would be essential to achieve better knowledge of $\mathrm{ATD}_{\mathrm{PHE} / \mathrm{LEU}}$ 's metabolic consequences. In this study, we chose the assessment of the BCAAs within a sum parameter for initial investigations as it provides a costsaving alternative. Moreover, a further aspect that should be considered in future studies would be to add caffeine withdrawal as an additional exclusion criterion since caffeine has been shown to induce alterations in serotonergic neurotransmission $(63,64)$.

The ATD method as a serotonergic challenge tool was criticized by Van Donkelaar et al. in a review article (65). Specifically, the authors advised caution in interpreting the selective serotonergic effect of ATDrelated results, postulating a contribution by various other non-serotonergic mechanisms to diminished 5-HT metabolism (e.g. alterations in cerebrovascular blood flow, stress influence, and metabolic effects). Furthermore, the missing possibility of direct attribution of ATD-induced behavioral alterations to changes in 5-HT neuronal activity was noted. However, many of the suggested mechanisms and the related hypotheses were based on limited data and primarily indirect conclusions. Overall, there is sufficient and convincing evidence that ATD leads to diminished central nervous 5-HT synthesis. In a rodent model, Biskup et al. (38) demonstrated substantially impaired brain 5-HT synthesis after ATD Moja-De administration. Furthermore, they found a lowered content of 5-HIAA, which is the primary metabolite of 5-HT, and a consequently reduced 5-HT release. As summarized and outlined by Crockett et al. (66), all of the alternative mechanisms suggested in the aforementioned review would lead to broad attentional and executive impairments that have not been found in any ATD study involving humans. In general, we support the need for further research to gain a solidly grounded understanding of the underlying mechanisms of ATD because the extent of contributions of additional mechanisms cannot be reliably estimated. 
In conclusion, the greater decline in TRP influx rates across the BBB in healthy adults after the dietary administration of the newly developed simplified ATD protocol, $\mathrm{ATD}_{\mathrm{PHE} / \mathrm{LEU}}$, relative to the established ATD Moja-De mixture, was the major finding of this study. The altered composition of $\mathrm{ATD}_{\mathrm{PHE} / \mathrm{LEU}}$, composed of only two LNAAs (PHE and LEU), enabled the reduction in the overall AA amount, had acceptable tolerance, and ensured simplified blood value monitoring due to the reduced components. Nevertheless, this work only represents a first pilot study. Future research is warranted not only to replicate the present findings (optimally a withinsubject repeated measures design) but also to improve beverage palatability, its specificity for central nervous 5-HT depletion, and to develop a more suitable balanced control condition (BAL $L_{\mathrm{PHE} / \mathrm{LEU}}$ ) without significant TRP-related depletion effects. The direct proof of reduced brain 5-HT synthesis in a rodent model (i.e. 5-HT concentrations in brain tissue) would be beneficial, as well as investigations of 5-HIAA levels in human cerebrospinal fluid in order to further validate the newly

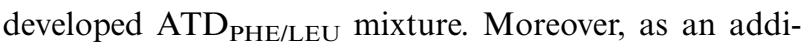
tional step, ATD $\mathrm{PHE}_{\mathrm{LEEU}}$ requires further study before administering this mixture to children and adolescents can be considered. If the remaining questions with regard to changes in plasma TRP levels, aspects of specificity, and the respective control condition are clarified, the simplified composition of ATD $\mathrm{PHE}_{\mathrm{PLE}}$ might have the potential to become an advantageous alternative to the currently available ATD Moja-De protocol for the investigation of the physiological and behavioral effects of reduced central nervous 5-HT synthesis in translational research. However, at this stage, the newly developed ATD PHE-LEU $_{\text {mixture needs further validation and }}$ is currently not suitable to specifically study central nervous 5-HT function in humans, and at this point in time it cannot replace the ATD Moja-De ATD protocol.

\section{Acknowledgements}

This study was funded by the German Federal Ministry of Economics and Technology (Bundesministerium für Wirtschaft und Technologie, BMWi) with a grant to the senior author of this article (FDZ). The lead author (ML) was supported by the German Research Council (Deutsche Forschungsgemeinschaft DFG, IRTG 1328).

\section{Conflict of interest and funding}

FDZ was the recipient of an unrestricted award donated by the American Psychiatric Association (APA), the American Psychiatric Institute for Research and Education (APIRE), and AstraZeneca (Young Minds in Psychiatry Award). He has also received research support from the German Federal Ministry for Economics and Technology, the European Union (EU), the German Society for Social Pediatrics and Adolescent Medicine, the Paul and Ursula
Klein Foundation, the Dr. August Scheidel Foundation, the IZKF fund of the University Hospital of RWTH Aachen University, the Telethon Perth Childrens' Hospital Research Fund (TPCHRF), the Princess Margaret Hospital Foundation, the Telethon Kids Institute, and a travel stipend donated by the GlaxoSmithKline Foundation. He is the recipient of an unrestricted educational grant, travel support, and speaker honoraria by Shire Pharmaceuticals, Germany. In addition, he has received support from the Raine Foundation for Medical Research (Raine Visiting Professorship) and editorial fees from Co-Action Publishing (Sweden). All authors report no conflicts of interest.

\section{References}

1. Zepf FD, Hood S, Guillemin GJ. Food and your mood: nutritional psychiatry. Lancet Psychiatry 2015; 2(7): e19.

2. Delgado PL, Charney DS, Price LH, Aghajanian GK, Landis H, Heninger GR. Serotonin function and the mechanism of antidepressant action. Reversal of antidepressantinduced remission by rapid depletion of plasma tryptophan. Arch Gen Psychiatr 1990; 47(5): 411-18.

3. Kishi T, Yoshimura R, Fukuo Y, Okochi T, Matsunaga S, Umene-Nakano $\mathrm{W}$, et al. The serotonin 1A receptor gene confer susceptibility to mood disorders: results from an extended metaanalysis of patients with major depression and bipolar disorder. Eur Arch Psychiatr Clin Neurosci 2013; 263(2): 105-18.

4. Kotting WF, Bubenzer S, Helmbold K, Eisert A, Gaber TJ, Zepf FD. Effects of tryptophan depletion on reactive aggression and aggressive decision-making in young people with ADHD. Acta Psychiatr Scand 2013; 128(2): 114-23.

5. Mette C, Zimmermann M, Grabemann M, Abdel-Hamid M, Uekermann J, Biskup CS, et al. The impact of acute tryptophan depletion on attentional performance in adult patients with ADHD. Acta Psychiatr Scand 2013; 128(2): 124-32.

6. Smith KA, Fairburn CG, Cowen PJ. Symptomatic relapse in bulimia nervosa following acute tryptophan depletion. Arch Gen Psychiatr 1999; 56(2): 171-6.

7. Zimmermann M, Grabemann M, Mette C, Abdel-Hamid M, Uekermann J, Kraemer M, et al. The effects of acute tryptophan depletion on reactive aggression in adults with attention-deficit/ hyperactivity disorder (ADHD) and healthy controls. PLoS One 2012; 7(3): e32023.

8. Biskup CS, Gaber T, Helmbold K, Bubenzer-Busch S, Zepf FD. Amino acid challenge and depletion techniques in human functional neuroimaging studies: an overview. Amino Acids 2015; 47(4): 651-83.

9. Helmbold K, Zvyagintsev M, Dahmen B, Bubenzer-Busch S, Gaber TJ, Crockett MJ, et al. Effects of serotonin depletion on punishment processing in the orbitofrontal and anterior cingulate cortices of healthy women. Eur Neuropsychopharmacol 2015 ; 25(6): 846-56

10. Hildebrand P, Königschulte W, Gaber TJ, Bubenzer-Busch S, Helmbold K, Biskup CS, et al. Effects of dietary tryptophan and phenylalanine-tyrosine depletion on phasic alertness in healthy adults-a pilot study. Food Nutr Res 2015; 59: 26407.

11. Sanchez CL, Biskup CS, Herpertz S, Gaber TJ, Kuhn CM, Hood SH, et al. The role of serotonin (5-HT) in behavioral control: findings from animal research and clinical implications. Int J Neuropsychopharmacol 2015; 18(10): pyv050.

12. Sánchez CL, Van Swearingen AE, Arrant AE, Biskup CS, Kuhn CM, Zepf FD. Simplified dietary acute tryptophan 
depletion: effects of a novel amino acid mixture on the neurochemistry of C57BL/6J mice. Food Nutr Res 2015; 59: 27424.

13. Gaber TJ, Dingerkus VL, Crockett MJ, Bubenzer-Busch S, Helmbold K, Sánchez CL, et al. Studying the effects of dietary body weight adjusted acute tryptophan depletion on punishment-related behavioral inhibition. Food Nutr Res 2015; 59: 28443.

14. Pardridge WM, Oldendorf WH. Kinetic analysis of blood-brain barrier transport of amino acids. Biochim Biophys Acta 1975; 401(1): 128-36.

15. Oldendorf WH, Szabo J. Amino acid assignment to one of three blood-brain barrier amino acid carriers. Am J Physiol 1976; 230(1): 94-8.

16. Smith QR, Momma S, Aoyagi M, Rapoport SI. Kinetics of neutral amino acid transport across the blood-brain barrier. J Neurochem 1987; 49(5): 1651-8.

17. Smith QR, Takasato Y. Kinetics of amino acid transport at the blood-brain barrier studied using an in situ brain perfusion technique. Ann N Y Acad Sci 1986; 481: 186-201.

18. Moja EA, Restani P, Corsini E, Stacchezzini MC, Assereto R, Galli CL. Cycloheximide blocks the fall of plasma and tissue tryptophan levels after tryptophan-free amino acid mixtures. Life Sci 1991; 49(15): 1121-8.

19. Wurtman RJ, Hefti F, Melamed E. Precursor control of neurotransmitter synthesis. Pharmacol Rev 1980; 32(4): 315-35.

20. Marsh DM, Dougherty DM, Moeller FG, Swann AC, Spiga R. Laboratory-measured aggressive behavior of women: acute tryptophan depletion and augmentation. Neuropsychopharmacology 2002; 26(5): 660-71.

21. Dougherty DM, Marsh-Richard DM, Mathias CW, Hood AJ, Addicott MA, Moeller FG, et al. Comparison of 50- and 100-g L-tryptophan depletion and loading formulations for altering 5-HT synthesis: pharmacokinetics, side effects, and mood states. Psychopharmacology (Berl) 2008; 198(3): 431-45.

22. Fernstrom JD. Role of precursor availability in control of monoamine biosynthesis in brain. Physiol Rev 1983; 63(2): 484-546.

23. Chanut E, Zini R, Trouvin JH, Riant P, Tillement JP, Jacquot C. Albumin binding and brain uptake of 6-fluoroDL-tryptophan: competition with L-tryptophan. Biochem Pharmacol 1992; 44(10): 2082-5.

24. Frazer A, Hensler JG. Serotonin. In: Siegel GJ, Agranoff BW, Albers RW, Molinoff PB, eds. Basic neurochemistry: molecular, cellular and medical aspects. New York: Raven Press; 1994, pp. 283-308.

25. Marsden CA. The neuropharmacology of serotonin in the central nervous system. In: Feighner JP, Boyer WF, eds. Selective serotonin re-uptake inhibitors. Chichester: Wiley; 1996, pp. 1-34.

26. Dingerkus VL, Gaber TJ, Helmbold K, Bubenzer S, Eisert A, Sanchez CL, et al. Acute tryptophan depletion in accordance with body weight: influx of amino acids across the blood-brain barrier. J Neural Transm (Vienna) 2012; 119(9): 1037-45.

27. Zepf F. Principles of rapid tryptophan depletion and its use in research on neuropsychiatric disorders. In: D'Mello J, ed. Amino acids in human nutrition and health. Oxfordshire: Cabi; 2012, pp. 418-26.

28. Concu A, Fadda F, Blanco S, Congia S, Lostia M. Mental changes induced by the oral administration of tryptophan-free amino acid mixtures in man. IRCS Med Sci 1977; 5: 520

29. Fadda F. Tryptophan-free diets: a physiological tool to study brain serotonin function. News Physiol Sci 2000; 15: 260-4.

30. Moja EA, Stoff DM, Gessa GL, Castoldi D, Assereto R, Tofanetti O. Decrease in plasma tryptophan after tryptophanfree amino acid mixtures in man. Life Sci 1988; 42(16): 1551-6.
31. Young SN, Smith SE, Pihl RO, Ervin FR. Tryptophan depletion causes a rapid lowering of mood in normal males. Psychopharmacology 1985; 87(2): 173-7.

32. Booij L, Van der Does AJ, Haffmans PM, Spinhoven P, McNally RJ. Acute tryptophan depletion as a model of depressive relapse: behavioural specificity and ethical considerations. Br J Psychiatr 2005; 187: 148-54

33. Allen PP, Cleare AJ, Lee F, Fusar-Poli P, Tunstall N, Fu CH, et al. Effect of acute tryptophan depletion on pre-frontal engagement. Psychopharmacology 2006; 187(4): 486-97.

34. Klaassen T, Riedel WJ, Deutz NE, Van Someren A, Van Praag HM. Specificity of the tryptophan depletion method. Psychopharmacology 1999; 141(3): 279-86.

35. Moeller FG, Dougherty DM, Swann AC, Collins D, Davis CM, Cherek DR. Tryptophan depletion and aggressive responding in healthy males. Psychopharmacology 1996; 126(2): 97-103.

36. Kewitz A. Biochemische Untersuchungen zur Optimierung des 'Rapid Tryptophan Depletion-Test' (RTD): Eine physiologische Methode zur akuten Verminderung der zentrainervösen Serotonin-Synthese in der psychobiologischen Forschung. Johann Wolfgang Goethe-Universität zu Frankfurt am Main; 2002.

37. Demisch L, Kewitz A, Schmeck K, Sadigorsky S, Barta S, Dierks T, et al. Methodology of rapid tryptophan depletion (RTD): impact of gender and body weight. Eur Arch Psychiatry Clin Neurosci 2002; 252(2): 1.

38. Biskup CS, Sanchez CL, Arrant A, Van Swearingen AE, Kuhn C, Zepf FD. Effects of acute tryptophan depletion on brain serotonin function and concentrations of dopamine and norepinephrine in $\mathrm{C} 57 \mathrm{BL} / 6 \mathrm{~J}$ and $\mathrm{BALB} / \mathrm{cJ}$ mice. PLoS One 2012; 7(5): e35916.

39. Demal U. SKIDPIT-light Screeningbogen. Universität Wien; 1999.

40. Weiß R. Grundintelligenztest skala 2 - revision CFT 20-R [Culture fair intelligence test scale 2 - revision]. Göttingen: Hogrefe; 2006.

41. Zepf FD, Poustka F. 5-HT functioning and aggression in children with ADHD and disruptive behaviour disorders. Hum Psychopharmacol 2008; 23(5): 438.

42. Zepf FD, Wockel L, Poustka F, Holtmann M. Dietary tryptophan depletion according to body weight - a new treatment option in acute mania? Med Hypotheses 2009; 72(1): 47-8.

43. Zepf FD, Stadler C, Demisch L, Schmitt M, Landgraf M, Poustka F. Serotonergic functioning and trait-impulsivity in attention-deficit/hyperactivity-disordered boys (ADHD): influence of rapid tryptophan depletion. Hum Psychopharmacol 2008; 23(1): 43-51.

44. Zepf F, Holtmann M, Stadler C, Demisch L, Schmitt M, Wöckel L, et al. Diminished serotonergic functioning in hostile children with ADHD: tryptophan depletion increases behavioural inhibition. Pharmacopsychiatry 2008; 41(2): 60-5.

45. Pardridge WM. Brain metabolism: a perspective from the blood-brain barrier. Physiol Rev 1983; 63(4): 1481-535.

46. Helmbold K, Bubenzer S, Dahmen B, Eisert A, Gaber TJ, Habel U, et al. Influence of acute tryptophan depletion on verbal declarative episodic memory in young adult females. Amino Acids 2013; 45(5): 1207-19.

47. Watson D, Clark LA, Tellegen A. Development and validation of brief measures of positive and negative affect: the PANAS scales. J Pers Soc Psychol 1988; 54(6): 1063-70.

48. aan het Rot M, Benkelfat C, Boivin DB, Young SN. Bright light exposure during acute tryptophan depletion prevents a lowering of mood in mildly seasonal women. Eur Neuropsychopharmacol 2008; 18(1): 14-23.

49. Stadler C, Zepf FD, Demisch L, Schmitt M, Landgraf M, Poustka F. Influence of rapid tryptophan depletion on 
laboratory-provoked aggression in children with ADHD. Neuropsychobiology 2007; 56(2-3): 104-10.

50. Zepf FD, Wockel L, Poustka F, Holtmann M. Diminished 5-HT functioning in CBCL pediatric bipolar disorder-profiled ADHD patients versus normal ADHD: susceptibility to rapid tryptophan depletion influences reaction time performance. Hum Psychopharmacol 2008; 23(4): 291-9.

51. Nishizawa S, Benkelfat C, Young SN, Leyton M, Mzengeza S, de Montigny C, et al. Differences between males and females in rates of serotonin synthesis in human brain. Proc Natl Acad Sci USA 1997; 94(10): 5308-13.

52. Badawy AA, Dougherty DM, Richard DM. Specificity of the acute tryptophan and tyrosine plus phenylalanine depletion and loading tests part II: normalisation of the tryptophan and the tyrosine plus phenylalanine to competing amino acid ratios in a new control formulation. Int J Tryptophan Res 2010; 3: 35-47.

53. Riedel WJ, Klaassen T, Deutz NE, Van Someren A, Van Praag HM. Tryptophan depletion in normal volunteers produces selective impairment in memory consolidation. Psychopharmacology 1999; 141(4): 362-9.

54. Schmitt JA, Jorissen BL, Sobczak S, Van Boxtel MP, Hogervorst E, Deutz NE, et al. Tryptophan depletion impairs memory consolidation but improves focussed attention in healthy young volunteers. J Psychopharmacol 2000; 14(1): 21-9.

55. Sobczak S, Riedel WJ, Booij I, Aan Het Rot M, Deutz NE, Honig A. Cognition following acute tryptophan depletion: difference between first-degree relatives of bipolar disorder patients and matched healthy control volunteers. Psychol Med 2002; 32(3): 503-15.

56. Evers EA, Sambeth A, Ramaekers JG, Riedel WJ, Van der Veen FM. The effects of acute tryptophan depletion on brain activation during cognition and emotional processing in healthy volunteers. Curr Pharm Des 2010; 16(18): 1998-2011.

57. Young SN. Acute tryptophan depletion in humans: a review of theoretical, practical and ethical aspects. J Psychiatry Neurosci 2013; 38(5): 294-305.

58. Young SN, Leyton M. The role of serotonin in human mood and social interaction. Insight from altered tryptophan levels. Pharmacol Biochem Behav 2002; 71(4): 857-65.
59. Booij L, Van der Does W, Benkelfat C, Bremner JD, Cowen PJ, Fava M, et al. Predictors of mood response to acute tryptophan depletion. A reanalysis. Neuropsychopharmacology 2002; 27(5): 852-61.

60. Wieser H, Belitz HD. Relations between structure and bitter taste of amino acids and peptides. I. Amino acids and related compounds]. Z Lebensm Unters Forsch 1975; 159(2): 65-72.

61. Yudkoff M. Brain metabolism of branched-chain amino acids. Glia 1997; 21(1): 92-8.

62. Hutson SM, Lieth E, LaNoue KF. Function of leucine in excitatory neurotransmitter metabolism in the central nervous system. J Nutr 2001; 131(3): 846s-50s.

63. Khaliq S, Haider S, Naqvi F, Perveen T, Saleem S, Haleem DJ. Altered brain serotonergic neurotransmission following caffeine withdrawal produces behavioral deficits in rats. Pak J Pharm Sci 2012; 25(1): 21-5.

64. Haleem DJ, Yasmeen A, Haleem M, Zafar A. 24h withdrawal following repeated administration of caffeine attenuates brain serotonin but not tryptophan in rat brain: implications for caffeine-induced depression. Life Sci 1995; 57(19): PL285-92.

65. Van Donkelaar EL, Blokland A, Ferrington L, Kelly PA, Steinbusch HW, Prickaerts J. Mechanism of acute tryptophan depletion: is it only serotonin? Mol Psychiatry 2011; 16(7): 695-713.

66. Crockett MJ, Clark L, Roiser JP, Robinson OJ, Cools R, Chase HW, et al. Converging evidence for central 5-HT effects in acute tryptophan depletion. Mol Psychiatry 2012; 17(2): 121-3.

\footnotetext{
*Florian Daniel Zepf

Centre and Discipline of Child and Adolescent Psychiatry, Psychosomatics and Psychotherapy

University of Western Australia

35 Stirling Highway (M56I)

Crawley WA 6009, Perth

Australia

Email: florian.zepf@uwa.edu.au
} 